\title{
Optimization, Validation and Initial Clinical Implications of a Luminex-based Immunoassay for the Quantification of Fragile X Protein from Dried Blood Spots
}

\section{Anna Boggs}

Cincinnati Children's Hospital Medical Center

\section{Lauren Schmitt}

Cincinnati Children's Hospital Medical Center

Richard McLane

Cincinnati Children's Hospital Medical Center

\section{Tatyana Adayev}

Giuseppe LaFauci

Kelli Dominick

Cincinnati Children's Hospital Medical Center

Christina Gross

Cincinnati Children's Hospital Medical Center

\section{Paul Horn}

Cincinnati Children's Hospital Medical Center

Craig Erickson ( $\square$ craig.erickson@cchmc.org )

Cincinnati Children's Hospital Medical Center

\section{Research Article}

Keywords: fragile X syndrome, biomarker, molecular phenotype

Posted Date: September 16th, 2021

DOI: https://doi.org/10.21203/rs.3.rs-850436/v2

License: (c) (1) This work is licensed under a Creative Commons Attribution 4.0 International License. Read Full License 
1 Optimization, validation and initial clinical implications of a Luminex-based immunoassay for the

2 quantification of Fragile X Protein from Dried Blood Spots

3 Anna E Boggs ${ }^{1}$, Lauren M Schmitt ${ }^{2,3}$, Richard D McLane ${ }^{1}$, Tatyana Adayev ${ }^{6}$, Giuseppe LaFauci ${ }^{6}$, Paul S

4 Horn $^{3,5}$, Kelli C. Dominick ${ }^{1,4}$, Christina Gross ${ }^{3,5}$, Craig A Erickson ${ }^{1,4, *}$

5 1. Division of Child and Adolescent Psychiatry (MLC 4002), Cincinnati Children's Hospital Medical

$6 \quad$ Center, 3333 Burnet Ave., Cincinnati, OH, 45229-3039, USA

7 2. Division of Developmental and Behavior Pediatrics, Cincinnati Children's Hospital Medical

8 Center, 3333 Burnet Ave., Cincinnati, OH, 45229-3039, USA

9 3. Department of Pediatrics, University of Cincinnati College of Medicine, Cincinnati, OH, USA

10 4. Department of Psychiatry, College of Medicine, University of Cincinnati, Cincinnati, OH, USA

11 5. Division of Neurology, Cincinnati Children's Hospital Medical Center, 3333 Burnet Ave.,

12 Cincinnati, OH, 45229-3039, USA

13 6. Department of Human Genetics, New York State Institute for Basic Research in Developmental

14 Disabilities, Staten Island, NY 10314, USA

15 *Corresponding author: Craig Erickson, 3333 Burnet Avenue, MLC 4002, Cincinnati, Ohio, 45229,

$16 \quad$ craig.erickson@cchmc.org

17

18

19

20

21

22 
Abstract (no more than 350 words)

Background: Fragile X syndrome (FXS) is the most common inherited form of intellectual disability affecting 1 in 4,000 males and 1 in 6-8,000 females. FXS is caused by a trinucleotide expansion

27 in the 5'UTR of the Fragile X Mental Retardation (FMR1) gene which in full mutation carriers (>200 repeats) leads to hypermethylation and transcriptional silencing of the gene and lack of expression of

29 Fragile X Protein (FXP, formerly known as Fragile X Mental Retardation Protein, FMRP). Phenotypic presentation of FXS is highly variable, and molecular markers explaining or predicting this variability are

31 lacking. Recent studies suggest that trace amounts of FXP can be detected even in fully methylated

32 individuals and may have clinical relevance; however, the lack of available reproducible, sensitive assays to detect FXP in peripheral tissue makes evaluation of peripheral FXP as a source of clinical variability challenging.

Methods: We optimized a Luminex-based assay to detect FXP in dried blot spots for increased reproducibility and sensitivity by improving reagent concentrations and buffer conditions. The optimized assay was used to quantify FXP in 187 individuals (101 males, 86 females; 0-78.4 years) including 35 typically developing controls (24 males, 11 females), 103 individuals carrying full mutations (70 males, 33 females), and 49 individuals with premutations ( 7 males, 42 females). A subset of these individuals showed repeat number or methylation mosaicism. We investigated the clinical relevance of peripheral

41 FXP levels by examining its relationship with general intellectual functioning in a subset of individuals 42 with available IQ scores.

Results: We show that the optimized assay is highly reproducible and detects a wide range of 44 FXP levels. Mosaic individuals had, on average, higher FXP levels than fully methylated individuals, and 45 trace amounts of FXP were consistently detectable in a subset of individuals with full mutation FXS. IQ 
scores were positively correlated with peripheral FXP levels in male and female individuals with full

47 mutation FXS.

Conclusions: We demonstrate that our optimized Luminex-based assay to detect FXP is reproducible, highly sensitive, and related to the core intellectual disability phenotype. Further, our data suggest that trace amounts of FXP detectable in dried blood spots of individuals with FXS could be

51 clinically relevant and may be used to stratify individuals with FXS for optimized treatment. Future

52 studies are needed with larger sample sizes, evaluating FXP across development and expanded analysis

53 of the relevance of FXP levels for behavioral and electrophysiological phenotypes in FXS.

\section{Background}

58 Fragile X Syndrome (FXS) is the most common single gene cause of autism spectrum disorder (ASD) and 59 most common inherited cause of intellectual disability impacting 1 in 4,000 males and 1 in 6-8,000

60 females worldwide (1). FXS results from CGG triplet repeat expansion in the promotor region of the

61 FMR1 gene located on the long arm of the $X$ chromosome (2). Typically, over 200 CGG repeats result in

62 gene methylation and transcriptional silencing of the FMR1 gene. The CGG repeats in the full mutation

63 range are typically inherited and undergo expansion when passed from a premutation carrier mother

64 (55-200 CGG repeats) to her child (2). As a disorder of gene silencing, FXS results from deficient

65 production of the FMR1 gene product, fragile $X$ protein (FXP, previously termed fragile $X$ mental

66 retardation protein (FMRP)) (3). FXP serves as a translational repressor impacting the expression of

67 hundreds of proteins vital to brain function including those critical to cognitive functioning (4). 
FXS is associated with a behavioral phenotype marked by high incidence of anxiety, ADHD,

69 language and cognitive deficits among other clinical features (5-7), and with physical presentations

70 including, but not limited to, pronounced ears, soft tissue laxity and macroorchidism in males (8).

71 Despite commonalities in the presentation, significant variation in the behavioral phenotype does exist

72 within FXS. Females with FXS are obligate mosaics with two X chromosomes resulting in a highly variable

73 phenotype in girls and women ranging from no appreciable developmental impairment to significant

74 development delay or intellectual disability. Even among males with FXS, phenotypic developmental

75 variability is represented by functioning levels from severe to mild or even borderline

76 intellectual/cognitive impairment (8). In part, this variation may be due to mosaicism in FXS. Repeat size

77 mosaicism can occur when individuals have a mix of premutation and full mutation repeat alleles,

78 whereas methylation mosaicism can occur where clinical Southern Blot and PCR testing may indicate

79 inconsistent methylation patterns with a mix of fully and non-fully methylated FMR1 alleles regardless

80

of CGG repeat length. Given the phenotypic and genetic variation in FXS, it is of critical importance to

81 understand how this variation may relate to variable FXP expression in this disorder.

83 sensitivity and specificity of available assays. In addition, there are limited studies assessing how well

84 FXP levels in accessible peripheral cells reflect FXP levels in the brain and whether FXP in peripheral cells

85 is associated with characteristic phenotypic features. Nevertheless, in recent years there has been

86 considerable progress in methodology for FXP detection and measurement in peripheral tissue such as

87 human blood, skin fibroblasts, hair follicles and buccal cells (9-14). One of the first attempts to evaluate

88 FXP levels used immunofluorescent staining in blood lymphocytes (15). This method was subsequently

89 used to document the significant relationship between higher FXP levels and higher cognitive

90 functioning based on IQ scores $(6,16,17)$. However, this assay did not take into account different

91 expression levels of FXP in individual lymphocytes and thus lacked sensitivity to detect a spectrum of 
92 FXP expression, which may be clinically relevant. Despite Western blot and ELISA (enzyme-linked

93 immunosorbent assay) methods providing a continuous readout that better captures the full range of

94 FXP expression, these methods are difficult to scale up (western blot) and lack quantifiability, sensitivity

95 and/or specificity (reviewed in (14)).

96 Recently, a novel highly sensitive assay was developed measuring FXP as a continuous variable

97 in peripheral blood using Luminex-based technology (18). This method is not only more sensitive to

detect lower values of FXP than previous methods, but also the Luminex assay is easily scalable and has

99 increased specificity due to the use of two different highly specific FXP antibodies and. Moreover, this

100 assay can be used with eluates from dried blood spots, which facilitates the potential future application

101 of this assay outside the research laboratory and into clinical settings. The FXP Luminex assay was used

102 in a recent study to measure FXP levels in 42 samples of individuals with FXS and demonstrated that

103 males with severe ID had lower FXP than males with mild or moderate ID (19). However, the lower limit

104 of FXP detection was above zero indicating difficulty differentiating "true zero" FXP expression from

105 potential low or trace level FXP levels, thus also limiting its capacity to be more clinically relevant among

106 males with full mutation.

Thus, given the major advantages the Luminex assay offered over previous methods to measure

108 FXP, we sought to optimize this method to improve upon the detection of FXP in peripheral blood to

109 best discern potential molecular variation in FXS. We believe a highly sensitive and reproducible FXP

110 assay will be important to new treatment development as FXP expression likely varies extensively in FXS

111 despite the single gene/single protein nature of the disorder. In appreciating molecular variation, we

112 aim to in the future use this understanding as a means to biologically subgroup persons with FXS when

113 evaluating clinical presentation, outcome, and response to potential therapeutics. Here, we report on

114 our initial work to optimize FXP detection in human blood with a focus on enhancing assay accuracy and 
115 improving the lower limit of detection using the FXP assay in broad subgroups of individuals with

116 emphasis on populations of males and females with full mutation FXS.

\section{Methods}

\section{Participants}

119 We enrolled a total of 187 participants: 101 males and 86 females aged $0-78$ years (Table 1).

120 Participants with a full mutation (70 males and 33 females) or premutation ( 7 males and 42 females) in

121 the FMR1 gene were recruited through the Cincinnati Fragile X Research and Treatment Center. Fragile X

122 status was confirmed at minimum by clinical Southern Blot (SB) and/or Polymerase Chain Reaction (PCR)

123 testing to confirm group assignment. Among individuals with full mutation FXS, 54 males and 29 females

124 had reliable research standard SB and PCR analysis completed at Rush University to evaluate for repeat

125 size mosaicism, methylation mosaicism, or expression of both mosaicisms. Within this subsample, 18

126 males (33.3\%) exhibited methylation and/or size mosaicism and 9 females (31\%) had methylation

127 mosaicism in addition to being obligate size mosaics. Control subjects ( 24 males and 7 females) were

128 recruited through web-based fliers from the local community and had no prior diagnosis or treatment

129 for developmental or neuropsychiatric disorders. All participants or their legal guardians gave written

130 informed consent and/or verbal assent, when appropriate. This project was approved by the CCHMC

131 IRB.

Table 1. Demographic Information. 


\begin{tabular}{|c|c|c|c|c|c|c|c|c|}
\hline & \multirow{2}{*}{ No. } & \multicolumn{3}{|c|}{ Age } & \multicolumn{4}{|c|}{ Fragile X Protein (FXP) Concentration (pM) } \\
\hline & & Average & SD & Range & Average & Median & SD & Range \\
\hline Male & 101 & 19.2 & 15.3 & $0.3-61.6$ & 10.2 & 1.7 & 13.4 & $0.0-43.2$ \\
\hline Typically Developing & 24 & 26.0 & 14.9 & $5.5-60.1$ & 28.9 & 27.5 & 6.4 & $18.1-43.2$ \\
\hline Premutation & 7 & 31.1 & 23.4 & $2.2-61.6$ & 30.5 & 26.1 & 7.9 & $22.6-42.1$ \\
\hline Fragile X Syndrome & 70 & 15.7 & 13.2 & $0.3-45.7$ & 1.7 & 0.5 & 2.6 & $0.0-10.3$ \\
\hline Nonmosaic & 36 & 18.2 & 14.6 & $0.3-45.7$ & 0.6 & 0.3 & 1.2 & $0.0-6.2$ \\
\hline Mosaic & 18 & 14.8 & 13.8 & $0.5-41.5$ & 3.8 & 2.6 & 3.2 & $0.0-10.3$ \\
\hline Female & 86 & 33.2 & 18.9 & $0.2-78.4$ & 26.4 & 24.8 & 10.6 & $3.1-64.9$ \\
\hline Typically Developing & 11 & 20.9 & 18.9 & $0.2-63.8$ & 31.6 & 27.5 & 13.7 & $16.1-64.9$ \\
\hline Premutation & 42 & 47.3 & 13.2 & $9.0-78.4$ & 30.1 & 28.5 & 9.6 & $11.8-55.2$ \\
\hline Fragile X Syndrome & 33 & 19.4 & 10.3 & $1.2-42.9$ & 19.9 & 20.9 & 7.1 & $3.1-33.8$ \\
\hline Methylation Mosaicism & 9 & 16.4 & 7.8 & $1.2-25.2$ & 22.0 & 20.9 & 7.7 & $9.3-30.6$ \\
\hline
\end{tabular}

\section{Blood Collection and Processing}

135 Blood samples were collected from all participants in $2 \mathrm{~mL}$ Vacutainer K2EDTA tubes (BD, 36781) and

136 inverted 10 times before processing to ensure homogeneity within the sample. $50 \mu \mathrm{L}$ of blood was

137 pipetted onto ID Bloodstain Cards (Whatman Bloodstain Cards, WB100014) producing at least two cards

138 with 13 spots each from one sample collection. Cards were dried and stored with desiccant packs in low-

139 gas-permeable bags (VWR, 89027-022) within 4-24 hours after spotting to ensure DBS stability, in

140 accordance with dried blood spot (DBS) guidelines and published protocols $(18,19)$ (Figure 1).

\section{Elution of DBS}

142 From each card, five $6.9 \mathrm{~mm}$ diameter disks were prepared using a hole punch and transferred into

143 CoStar Spin-X Centrifuge Filter Tubes (7200388). Proteins were extracted from the DBS using $333 \mu \mathrm{L}$ of

144 elution buffer (M-PER with salt, Antipain, Chymostatin, Protease Inhibitor) with orbital shaking overnight

145 at room temperature. The eluates were collected after a 6-minute centrifugation at $12000 \times \mathrm{xg}$ and

146 immediately used in the assay. $50 \mu \mathrm{L}$ of the eluate was used per well in the assay (Figure 1). 
148 An 11-point standard curve was created using a purified GST-SR7 fusion protein obtained from the 149 Institute for Basic Research in Developmental Disabilities (IBR). Elution buffer was used to complete a 150 two-fold dilution of the first standard point generating a standard curve with range of 70-0.07 pM. 151 Either $50 \mu \mathrm{L}$ of standard or DBS extract was aliquoted into assay wells of a 96-well low protein binding 152 plates (Greiner Bio-One, 655096). The capture antibody, mAb 6B8 (BioLegend, 834601), was 153 concentrated according to manufacturer's instructions (Abcam, ab102778). The concentrated mAb 6B8 154 was coupled to Luminex Magspheres according to manufacturer's instructions and constructed at a 155 stock concentration of $100 \mu \mathrm{L}$ antibody/all beads. Beads were diluted in assay buffer (PBS, $1 \%$ BSA, $1560.05 \%$ Tween) to 80 beads/ $\mu \mathrm{L}$ for use in assay. Diluted beads were added to the assay wells at a volume 157 of $50 \mu \mathrm{L}$ to bring the final well volume to $100 \mu \mathrm{L}$. Plates were then incubated at room temperature for 6 158 hours on a microplate shaker. A Luminex magnetic plate washer was used to manually wash the plates 159 in assay buffer. After washing, the plates were incubated overnight in secondary detecting antibody 160 (ab17722, Abcam) at a dilution of 1:1000 (v:v). Plates were vigorously washed and incubated at room 161 temperature for 2 hours in signal detecting antibody (Jackson ImmunoResearch, 711-116-152). Plates 162 were vigorously washed and resuspended in $100 \mu \mathrm{L}$ of sheath fluid (Luminex, 40-50021). The 163 magspheres were analyzed (in quintuplicate) on the Luminex 200 system to determine median 164 fluorescence intensity (Figure 1). 


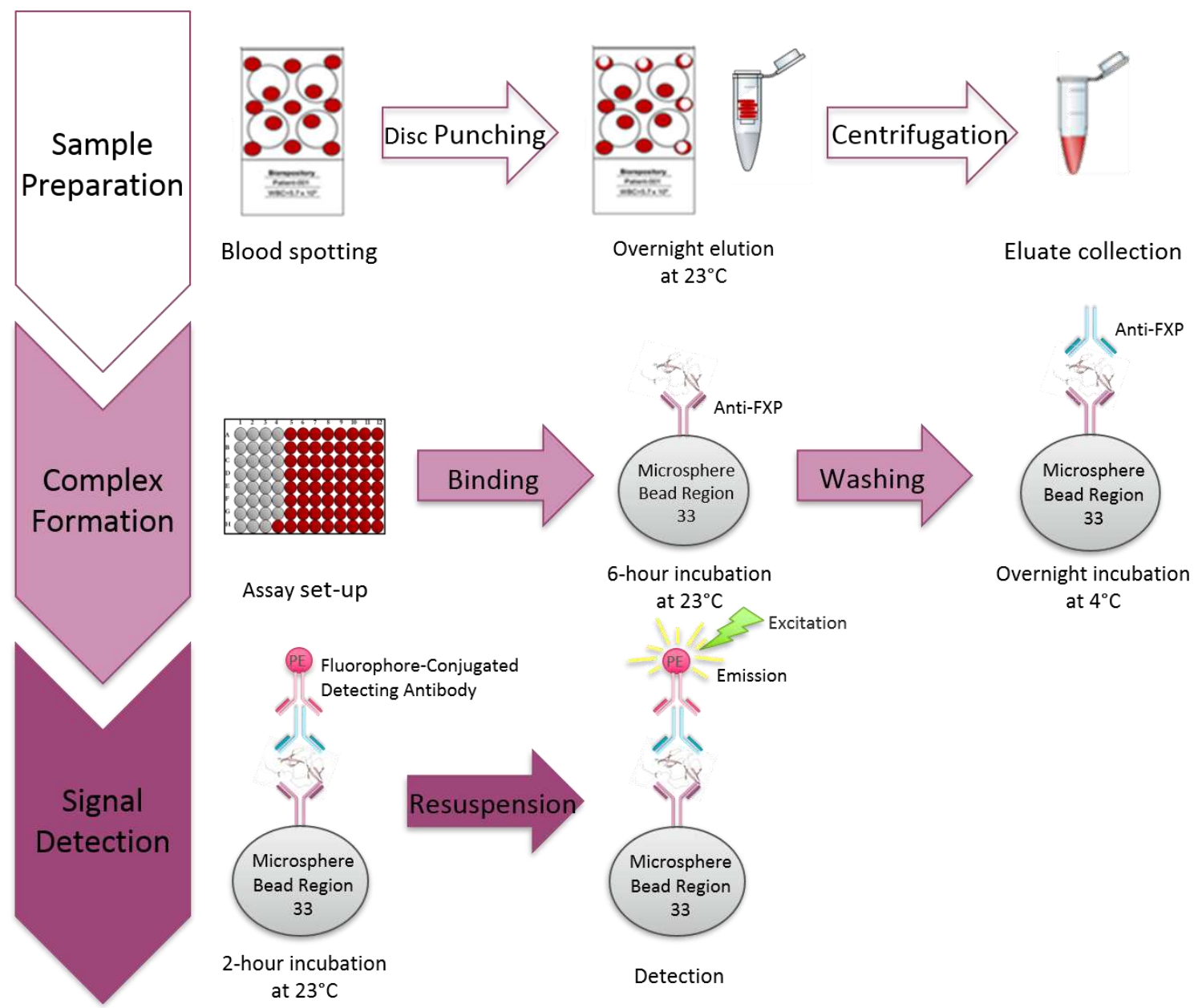

Figure 1. Experimental workflow of the optimized FXP assay.

Fragile X Protein (FXP) Quantification

168 To determine individual FXP concentration, BioPlex Manager Software was used to generate a standard 169 curve of GST-SR7 concentration as a function of median fluorescence intensity. Patient samples were 170 plotted against this curve and reported as concentration (pM) in the DBS extract. Based on the volume 171 of blood spotted, the size of the DBS, and the elution volume, we estimate peripheral FXP circulating in 172 the blood is approximately six times the value reported in the assay. Individual FXP concentrations were reported as a $20 \%$ trimmed mean of the observed

174 concentrations between the sample quintuplicates. The sample mean is prone to undue influence by 
175 extreme observations, while the sample median is inefficient if the data are normally distributed. The

$17620 \%$ trimmed mean, i.e., the average of the three central values, is used as a compromise between the

177 sample mean and sample median. Differences in protein concentration between groups were analyzed

178 using Kruskal-Wallis tests with Dunn's multiple comparison adjustment in GraphPad Prism software. A p-

179 value $<0.01$ was considered significant.

\section{Assay Reproducibility Analysis}

181 The stability of FXP over time was analyzed using the Intra-Class Correlation Coefficient (ICC) via SAS ${ }^{\circledR}$

182 software. Specifically, the agreement across time was measured using the ICC where the response, FXP,

183 was modeled as a function of subject ID and week. The Shrout-Fleiss measure of reliability, ICC, was

184 used where the subjects were evaluated at the various repeated measure timepoints (20). The ICC here

185 was the same regardless of whether the weeks were treated as a fixed or random variable.

186 To best characterize the assay, we completed rigorous testing of its performance statistics. We

187 measured intra-assay variability, inter-plate variability, inter-draw variability, and inter-card variability to

188 determine an acceptable standard of variation. Previous studies $(20,21)$ using the Luminex-based

189 immunoassay platform for FXP-unrelated assays have reported intrinsic variabilities and ranges of

190 acceptable variation. Based on those reports, we defined poor variation as CV >15\%, good and

191 acceptable variation as $\mathrm{CV}<15 \%$, and excellent variation as $\mathrm{CV}<5 \%$. For the calculation of the

192 performance statistics, all interpolated values were considered regardless of their acceptance as a

193 reported value (Figure 2). Intra-assay variability, or the variability between replicates, was measured and

194 reported as an average \%CV for each set of quintuplicates across all participant samples and plates.

\section{Outlier Removal Analysis}

196 We used the coefficient of variation (CV) as the standard measure of variability for this assay. Percent CV

197 was determined using the observed concentration calculated via the BioPlex Manager Software [i.e., 
$\mathrm{CV}=$ (standard deviation (of observed mean quintuplicates)/mean (of observed mean quintuplicates))

$199 * 100]$. Due to intrinsic variability within any biological test, we set a threshold for acceptable variation 200 within the quintuplicates from one sample as a CV $<15 \%$. This threshold is within the range of the 201 performance statistics of this assay and in alignment with similar methods $(20,21)$. If upon the initial 202 assay, a sample set of quintuplicates produced an observed concentration CV $<15 \%$, the result was 203 accepted and reported as the $20 \%$ trimmed mean of the observed concentration. If the results of the 204 initial assay indicated CV $\geq 15 \%$, the assay was repeated with a new set of 5 DBS from the original DBS 205 card, if possible. If less than 5 DBS were available, the largest amount of available DBS was used. If the 206 results of the repeated assay indicated $C V<15 \%$, the results of the second assay were accepted and 207 reported as the $20 \%$ trimmed mean of the observed concentration. If the $C V \geq 15 \%$ for both the initial 208 and repeated assays, both sets of quintuplicates were analyzed in the outlier removal program via SAS 209 software.

This final stage of outlier determination was used to retain as much data as possible while not

211 allowing outliers to adversely affect the overall result. Specifically, the generalized extreme Studentized 212 deviate approach was applied to each sample that was based on five DBS (21). This method allows for 213 the simultaneous detection of multiple outliers, in this case, two. Note that the position of up to two 214 outliers can be found at either or both extremes of the sample. Once the outlier(s) were removed from 215 the data set, a new CV was calculated with the remaining values. If the new CV $<15 \%$, the $20 \%$ trimmed 216 mean of the remaining values was accepted and reported. If no outliers were removed or the new CV $\geq$ $21715 \%$, the average of the $20 \%$ trimmed means from both assays was calculated and reported (Figure 2).

218 These samples (very few, see results section) were not removed as we could not exclude that the 219 observed values represent true variability. 


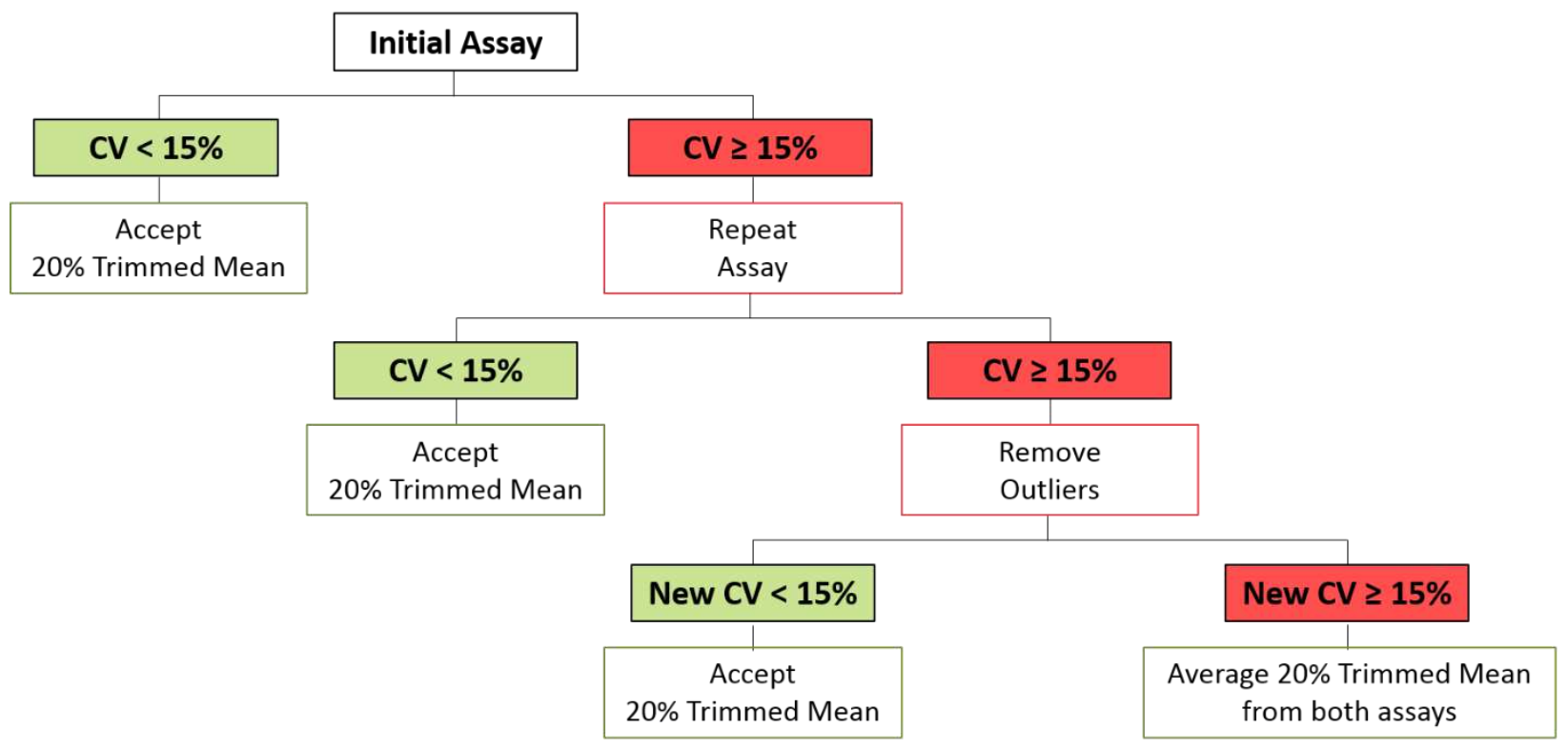

221 Figure 2. Result acceptance decision tree.

Correlation of Fragile X Protein Blood Level and Intellectual Function

In order to assess the potential clinical significance of FXP in blood as measured by our

225 optimized assay, we examined the linear and non-linear relationship between peripheral FXP and

226 intellectual functioning. For this, we examined a subset of participants with FXS ( $n=53$ ) who completed

227 the Abbreviated Battery of the Stanford-Binet, Fifth Edition (SB-5 (22)) as part of research evaluations

228 (Table 2). SB-5 full-scale IQ standard scores were converted to deviation scores to provide a better

229 estimate of intellectual ability in FXS participants $(22,23)$.

Table 2. Sub-set of Participants with FXS Included in IQ Correlation Analysis

\begin{tabular}{|l|l|l|l|l|l|l|}
\hline & \multicolumn{2}{|l|}{ Male } & \multicolumn{2}{l|}{ Female } & \multicolumn{2}{l|}{ Total } \\
\hline & FXS $(n=28)$ & TDC $(n=15)$ & FXS $(n=24)$ & TDC $(n=4)$ & FXS $(n=52)$ & TDC (n=19) \\
\hline Age & $29.9(8.8)$ & $27.8(8.3)$ & $21.8(8.6)^{*}$ & $17.3(1.3)$ & $26.2(9.5)$ & $25.6(8.6)$ \\
\hline
\end{tabular}




\begin{tabular}{|l|l|l|l|l|l|l|}
\hline Full-Scale IQ & $46.5(4.0)^{* * *}$ & $111.3(13.6)$ & $76.4(20.0)$ & $96.3(9.6)$ & $60.3(20.4)^{* * *}$ & $108.1(14.1)$ \\
\hline Deviation IQ & $32.2(17.1)^{* * *}$ & $108.9(13.0)$ & $77.5(18.1)$ & $93.6(8.9)$ & $53.1(28.7)^{* * *}$ & $105.7(13.6)$ \\
\hline
\end{tabular}

\section{Results}

\section{Immunoassay Optimization: Standard Curve and Lower Limit of Detection}

Unidentified components in whole blood can cause an overall decrease in signal intensity in

237 To test if the standard curve conditions used in the Luminex FXP assay accounted for the blood matrix

238 effect, we used dried blood spot eluates from two fully methylated (FM) FM males as the dilution buffer

239 for the standard protein, GST-SR7. We compared standard curves with this "blood buffer" against the

240 standard curve made with the Luminex buffer previously used $(15,22,23)$ and a standard curve made

241 with the elution buffer used for sample preparation (Figure 3). We observed that the blood components

242 globally decreased the median fluorescence intensity at each standard point compared with the

243 respective standard points prepared in Luminex buffer, suggesting that previously reported values may

244 have overestimated FXP in the blood. By contrast, the elution buffer closely mimicked the signal-

245 diminishing effect of the blood matrix. The patient blood curves showed variation in relative intensity,

246 most likely due to minute levels of endogenous FXP. Nevertheless, the elution buffer sufficiently

247 mimicked the signal-diminishing effects of the blood matrix while providing accurate representation of 248 sample preparation and was therefore used in this study. 


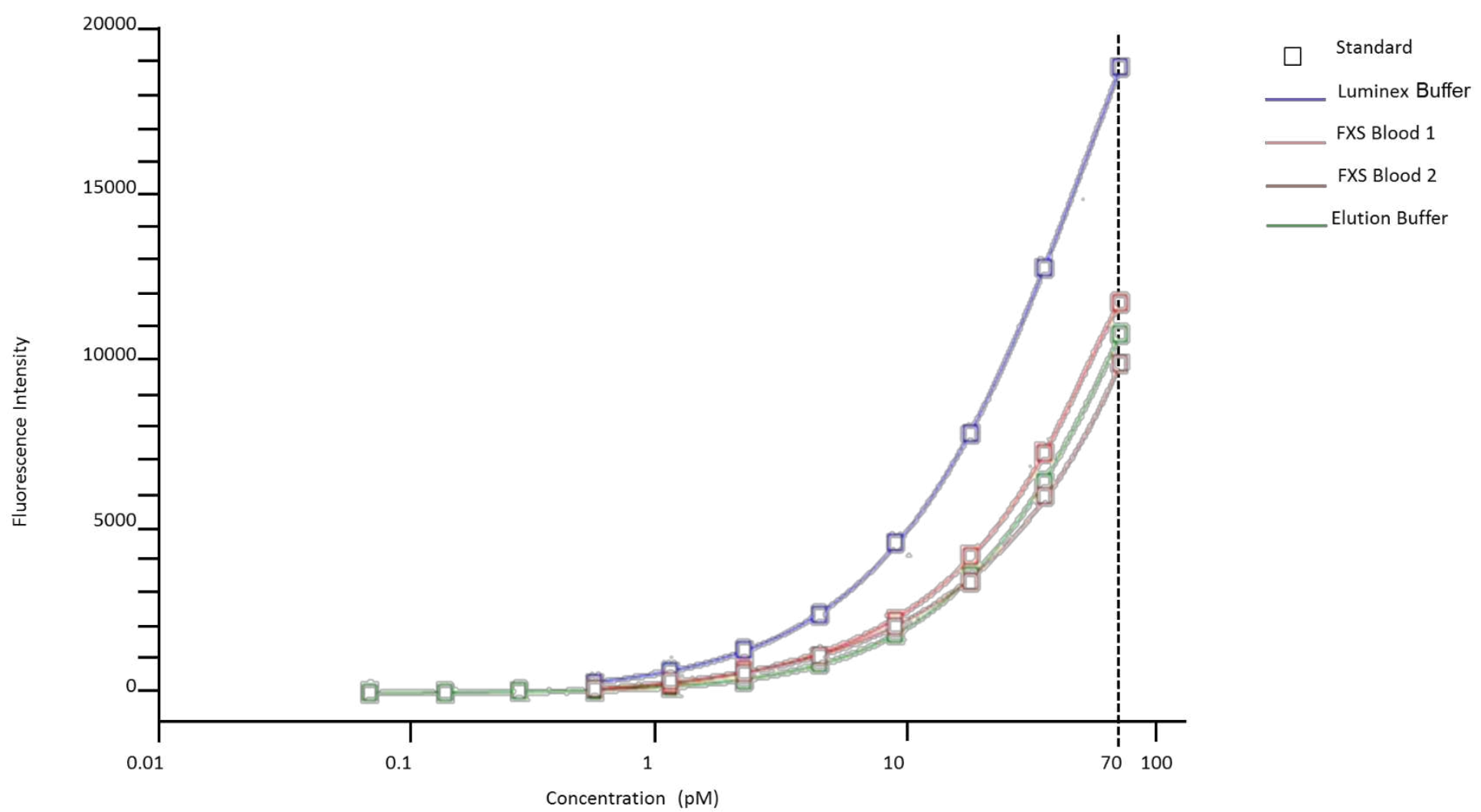

255

256

257

258

259

260

261

262

263

264

265

266

267

268

269

Figure 3. Elution buffer approximates blood matrix effect better than Luminex buffer. Luminex buffer (in blue) was previously used as the standard curve diluent. Due to components within the blood sample, demonstrated in the FXS blood curves (both red), fluorescent intensity is globally reduced. This blood matrix effect is mimicked by the elution buffer (in green), allowing the addition of more standard points to lower the detection limit to $0.07 \mathrm{pM}$.

We then evaluated the other components of the assay to negate any potential complications of interference or background due to the new buffer. Previous optimizations of ELISA-based methods (11, 27) utilized a checkerboard titration system to test multiple antibody conditions on the same test plate. Using this process, we compared different assay conditions to optimize magsphere preparation methods as well as compare two distinct detection antibodies, each at varying concentrations. The largest ratio of median fluorescence intensity between high and low standard points while maintaining a low background signal identified the optimized assay conditions. We determined that using a rabbit antihuman FXP (Abcam, ab17722) provided the highest signal-to-noise ratio with the optimized assay conditions (additional file 1). 
Since the optimized assay conditions decreased the overall background of the assay, we added

271 additional standard points to the low end of the standard curve which decreased the lower limit of

272 detection of the assay. We consistently and reliably have decreased the lower limit of detection to 0.07

273 pM (Figure 3). This allows for a more accurate and quantifiable measure for patients with low levels of

274 FXP which had been extrapolated, not quantified, using previous methods.

275 Analytical Validation of the Immunoassay

Intra-assay variability, or the variability between replicates, was measured and reported as an

277 average \%CV for each set of quintuplicates across all participant samples and plates. The intra-assay

278 variability could not be calculated for 119 out of $527(22.5 \%)$ of all quintuplicates because the values

279 were outside of the limits of detection and therefore unquantifiable, which was expected since

280 discernable FXP concentrations would not be expected for all FM FM males. The intra-assay variability

281 was poor for $19 \%$ of the quintuplicates while good and acceptable for the remaining $60 \%$ of assays $(47 \%$

282 of which had excellent variability). The overall average of the intra-assay variability (Table 3) was

283 congruent with similar methods $(11,20,21)$ and our threshold for outlier removal.

Table 3. Performance statistics of the optimized FXP Assay.

\begin{tabular}{|c|c|c|}
\hline Variability & Sample Size & \%CV \pm SD \\
\hline Intra-Assay & 553 (quintuplicates) & $10.1 \pm 7.9$ \\
\hline Inter-Plate & 41 (replications) & $9.4 \pm 10.2$ \\
\hline Inter-Draw & 26 (replications) & $6.0 \pm 4.1$ \\
\hline Inter-Card & 20 (replications) & $2.8 \pm 2.3$ \\
\hline
\end{tabular}

To determine the variability between plates (inter-plate variability), blood spots collected on the

287 same DBS card from one patient were analyzed concurrently on two plates. The assays were prepared 
288 independently of each other, using separate reagents and randomized analysis order. The inter-plate 289 variability was determined by calculating the CV between the $20 \%$ trimmed means from both assay

290 plates. The variability was poor for $15 \%$ of replications and good and acceptable for $70 \%$ of replications,

291 and the remaining $15 \%$ of replications were below the lower limit of detection so therefore the $\% \mathrm{CV}$

292 could not be calculated. The overall average inter-plate variability was congruent with the intra-assay

293 variability (Table 3).

294 Since one K2EDTA collection tube contains enough blood to produce two DBS cards, we

295 determined the inter-card variability, or the variability between both cards from the same blood draw.

296 This value was determined by analyzing each card in quintuplicate on the same assay, prepared with the

297 same conditions applied to each well. Some replications (25\%) that were below the lower limit of

298 detection and thus not quantifiable. This average inter-card variability was lower than the overall

299 variability (Table 3).

300 Strategy for Outlier Removal

301 The outlier removal analysis was only necessary for 47 quintuplicate samples (out of 527 total sample 302 runs, i.e. 8.9\%)). Upon completion of the repeated assay, 33 of the samples had acceptable CVs. The 303 remaining 14 repeated samples underwent outlier removal with 9 samples having successful removal of 304 outliers. Only 4 ( 1 male and 1 female TDC, 2 fully methylated full mutation (FM FM) males with FXS) 305 samples had a new $C V \geq 15 \%$ and thus have reported values of the average $20 \%$ trimmed means from 306 both assays. 
With the optimized assay conditions, we quantified peripheral FXP in DBS eluate from a total of

187 individuals across the diagnostic categories. Values are reported as a concentration (pM) of FXP in the DBS extract.

We first evaluated the expression of FXP between the diagnostic categories. FXP levels are significantly reduced in individuals with FXS when compared with premutation carriers and TDCs respectively. There was no significant difference in protein concentration between premutation carriers and TDCs (Figure 4A). We then analyzed the effects of sex on FXP. There was no significant difference between sex within the premutation carrier category nor within the TDCs (data not shown). However, as expected, there was a significant reduction in FXP in males with FXS in comparison to females with FXS (Mann-Whitney $\mathrm{U}=20, \mathrm{n}_{1}=70 \mathrm{n}_{2}=33, p<0.0001$ two-tailed) (Figure 4B).

A

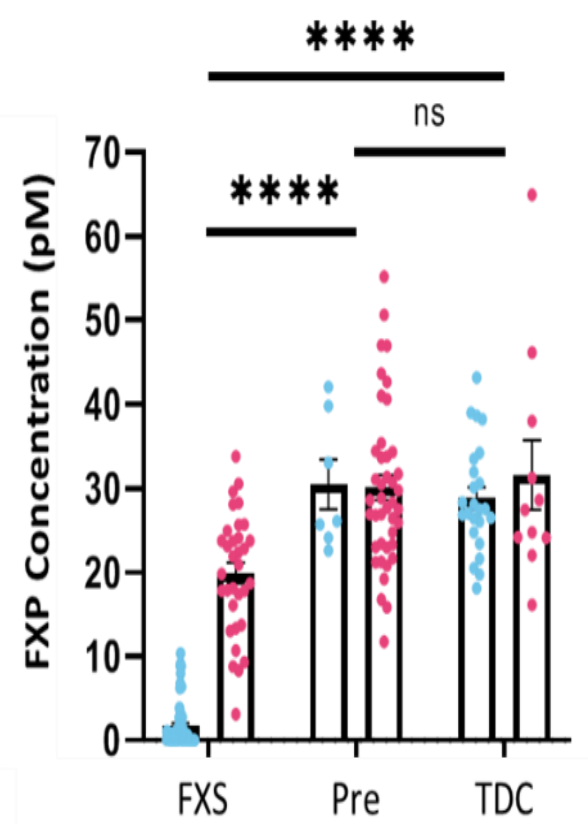

B

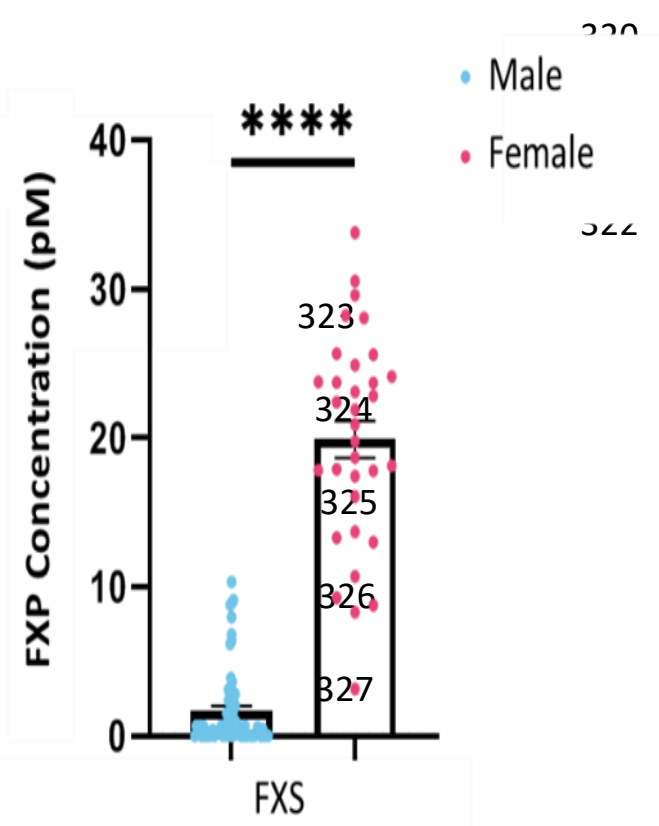

Figure 4. FXP concentration is reduced in FXS. (A) FXP concentration in all individuals across diagnostic categories from dried spot eluate. Graphically males and females are separated into individual bars; however, they were grouped as a diagnostic category when analyzed statistically. FXP concentration is significantly lower in the FXS diagnostic group than premutation carriers and TDC diagnostic groups (Kruskal-Wallis analysis with Dunn's multiple comparisons; $\mathrm{H}(3)=107.2,, p$ (FXS vs Pre) $<0.0001, p$ (FXS vs Pre) $<0.0001$ ). (B) FXP concentrations are significantly lower in males with FXS than females with FXS (Mann-Whitney $U=20, n_{1}=70 n_{2}=33, p<0.0001$ two-tailed). Mean reported with error bars representing SEM 
340 compared groups within sexes individually. Males with FXS had significantly lower FXP than their

341 premutation carrier and TDC counterparts. There was no difference between premutation males and

342 TDC males (Figure 5A). We observed the same trend in the females; females with FXS had significantly

343 lower FXP than premutation carriers and typically developing controls, though there was no difference

344 in FXP concentration between female premutation carriers and female typically developing controls

345 (Figure 5B). 
Male

A

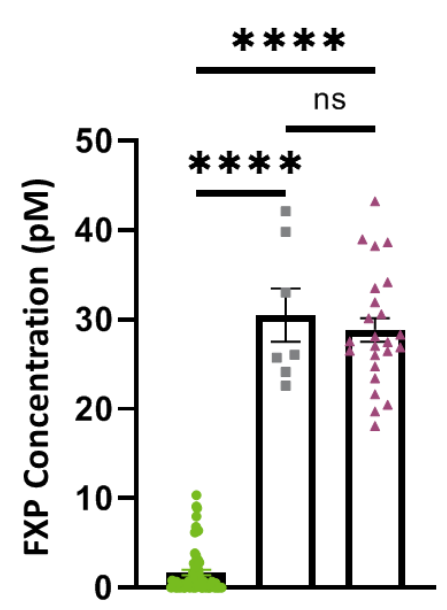

C

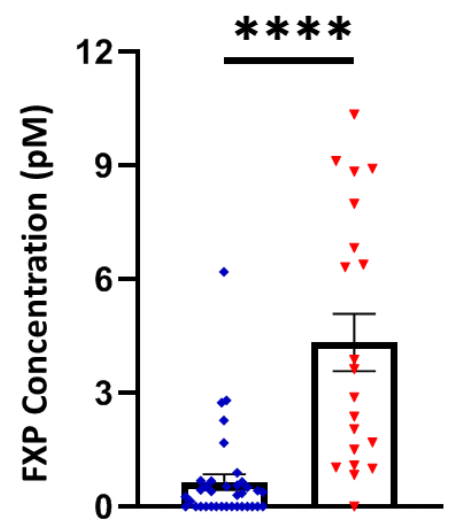

$\mathbf{E}$

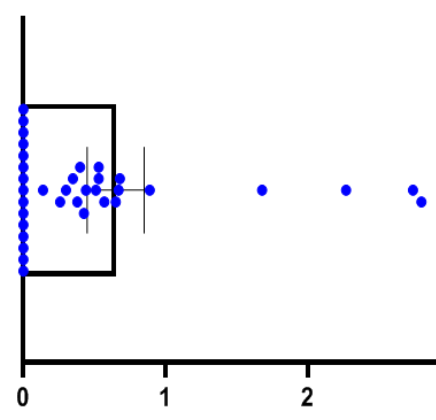

Female

B
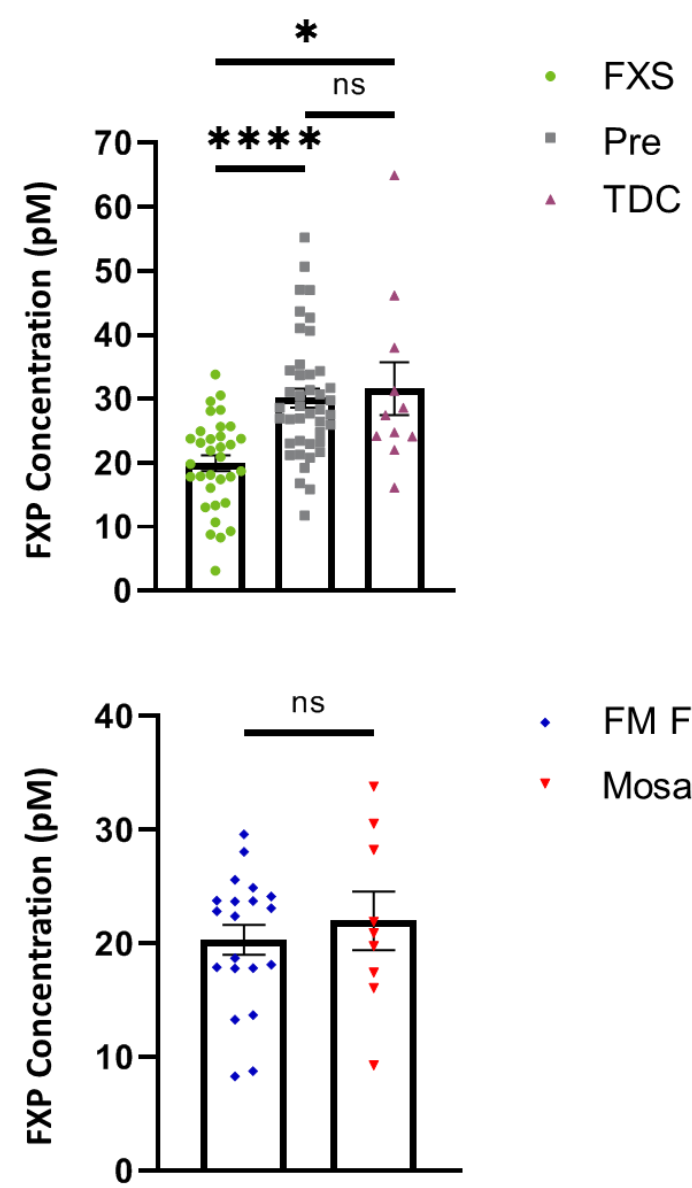

347 Figure 5. FXP concentration is reduced in males and females with FXS. (A) Males with FXS have significantly reduced FXP concentrations than premutation carriers $(p<0.0001)$ and typically developing controls (Kruskal-Wallis analysis with Dunn's multiple comparisons; $\mathrm{H}(3)=64.40, p$ (FXS vs Pre) <0.0001, $p$ (FXS vs Pre) < 0.0001). In our sample, there is no significant difference between FXP concentrations in premutation carriers and typically developing controls (Kruskal-Wallis analysis with Dunn's multiple comparisons; $\mathrm{H}(3)=64.40, p>0.9999)$. (B) Females with FXS had significantly lower FXP than premutation carriers and typically developing controls (Kruskal-Wallis analysis with Dunn's multiple 
comparisons; $\mathrm{H}(3)=22.12, p($ FXS vs Pre $)<0.0001, p($ FXS vs TDC $)=0.0101)$. There was no significant difference between female premutation carriers and TDCs (Kruskal-Wallis analysis with Dunn's multiple comparisons; $\mathrm{H}(3)=22.12, p>0.9999$ ). Not that data shown in A and $B$ are the same as in 4A. (C) FM FM males have significantly lower FXP concentration than males expressing mosaicism (Mann-Whitney $\mathrm{U}=68.5, \mathrm{n}_{1}=36 \mathrm{n}_{2}=20, p<0.0001$ two-tailed). (D) There is no significant difference between FM FM females and females with methylation mosaicism (Mann-Whitney $\mathrm{U}=84, \mathrm{n}_{1}=20 \mathrm{n}_{2}=9, p=0.7992$ twotailed). (E) Higher resolution of data for FM FM males illustrates that they express varying levels of FXP, ranging from undetectable to over 6 . Mean reported with error bars representing SEM

Next, we compared males that express the fully methylated (FM) FM to their mosaic male counterparts; here, mosaicism is a grouped category where individuals with either repeat or methylation mosaicism were analyzed as the mosaic group. As expected, FM FM males have significantly lower FXP than mosaic males. Interestingly, we found that there are some FM FM males that consistently express low amounts of FXP, but still less than the average mosaic male FXP level (Figure 5C). Since females are obligate mosaics due to their compensatory $X$ chromosome, we defined mosaicism for females based on methylation status alone. Using this definition, we differentiated between FM FM females and mosaic females that express both full mutation and premutation bands after southern blot analysis. There was no significant difference between FM FM females and methylation mosaic females (Figure 5D).

\section{Validation of Assay Reproducibility}

We evaluated within subject test-retest reproducibility of blood FXP in two cohorts, a group of 12 adults with full mutation FXS with FXP levels measured every two weeks totaling seven FXP samples and in 25 persons sampled less frequently (a minimum of two FXP measurements per patient; duration between assays 0.5 to 30 months). In both the short-term (Figure 6A) and longer-term within subjects repeated FXP testing (Figure 6B), the FXP results showed excellent intra-individual stability (ICC values were 98.8 and 97.6 , respectively). The average inter-draw variability was lower than the overall assay variability (Table 3). 
A

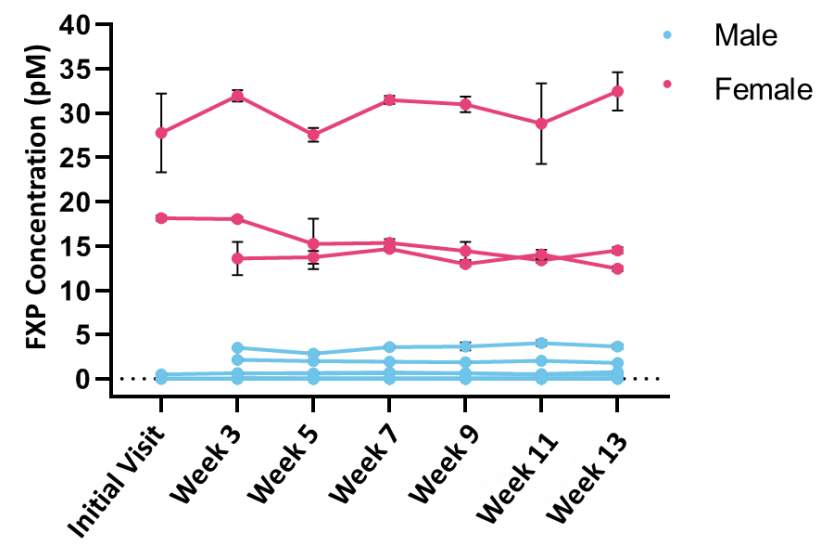

B

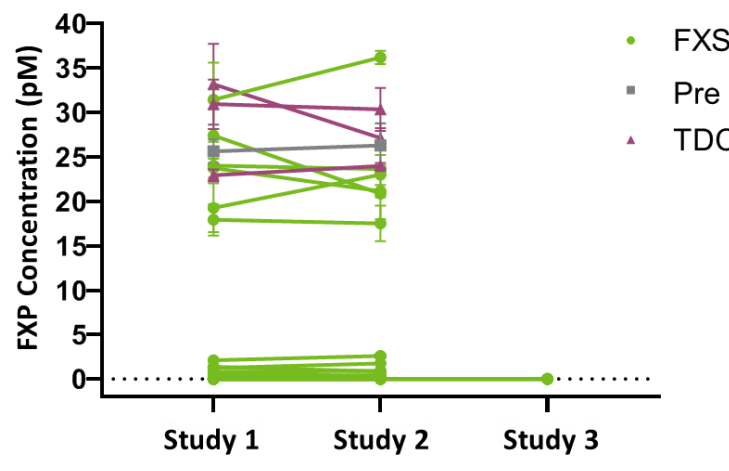
males with FXS, a linear model remained the best fit. In contrast, among females with FXS, logarithmic

Figure 6. FXP concentrations are consistent over time. (A) Repeated bi-weekly FXP levels in 12 adults with FM FXS (9 males; 3 females) showed consistency over 13 weeks (ICC value 98.8) (B) Over longer intervals between FXP testing (mean 11 months; range 0.5- 30 months), within subject FXP levels showed consistency (ICC value 97.6) in 25 persons (18 males, 7 females; age range $0.5-49.8$ years) with FXS, premutation, or no FX-related finding. Note that study 3 included multiple FM FM males with undetectable FXP levels.

\section{Correlation of FXP levels with intellectual function}

In both males $\left(r_{L I N}=.38 p=.04\right)$ and females $\left(r_{L I N}=.53, p=.01\right)$ with full mutation FXS, higher FXP concentrations were associated with higher Deviation IQ scores. However, when removing six male participants who had either size or methylation mosaicism, this relationship was no longer significant for males with FXS $(r=.15, p=.28)$. We next examined males with mosaicism and females together, with the thought that because mosaicism confers some production of FXP, the underlying mechanism supporting this relationship with intellectual functioning may be more similar to females with FXS than FM-FM. Deviation IQ and FXP remained significantly related $\left(r_{\mathrm{LIN}}=.73 p<.001\right)$.

Previous studies have indicated a non-linear model best describes the relationship between FXP and IQ in FXS (ref). Here, we conducted multiple non-linear models to determine which non-linear function best fit our data separately for males (Figure 7a) and females (Figure 7b) with FXS. Among

401 ( $\left.r_{\text {LOG }}=.56, r^{2}=.31, p=.005\right)$ and inverse $\left(r_{\mathrm{INV}}=.56, \mathrm{r}^{2}=.31, \mathrm{p}=.005\right)$ functions also fit the data. 

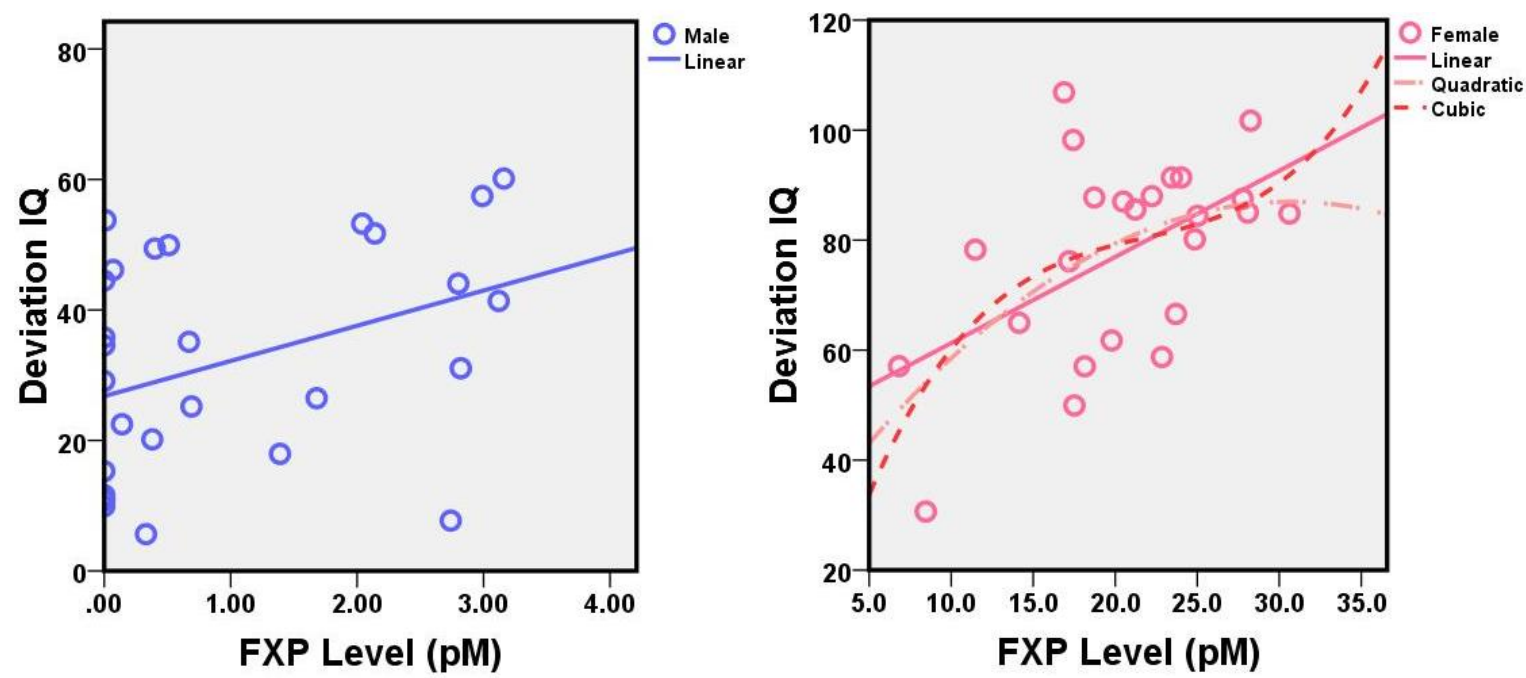
potential protein marker of disease in this field. Thus, the development of a continuous, reliable, and

414 clinically relevant biological marker of fragile X pathophysiology is of critical importance to translational

415 treatment development in this field. Such a marker would have potential clinical utility to predict patient subgroups that may best respond to treatment while also potentially serving as a future moveable

417 biological target of treatment itself. Our current work demonstrates that FXP as measured in peripheral 418 blood by our optimized assay holds promise as a reliable and clinically relevant biological marker in the 419

Figure 7. Linear and non-linear correlations between FXP concentrations and Deviation IQ scores for males (A) and females (B) with FXS

Next, due to the discrepancy in FXP concentration levels between males and females with FXS,

we normalized the data with log10 function in order to further assess relationship to IQ with the larger

FXS sample. We found significant relationships with linear $\left(r=.69, r^{2}=.46, p<.001\right)$, quadratic $(r=.83$,

$\left.r^{2}=.68, p<.001\right)$, and cubic $\left(r=.83, r^{2}=.69, p<.001\right)$ functions.

\section{Discussion}

Given FXS is defined at the core as a protein deficiency disorder, study of FXP is the most directly linked FXS field. 
Previous methods of quantifying FXP have had low signal to noise ratios that increase at the

421 lower limit of quantification and therefore mask potential low level protein expression (19). Although

422 these previous methods have shown initial clinical relevance by documenting relationships with general

423 cognitive ability, due to the restricted range of FXP expression captured by these methods, the full scope

424 of its clinical utility is limited (19). In the current study, using several key assay optimization techniques,

425 including standard curve and reagent optimization, we have developed a reliable and reproducible

426 means to quantify FXP in blood using blood spot cards. Using extensive test-retest strategies we have

427 validated reproducibility of this assay across a wide range of FXP values. In conjunction with lowering

428 the limit of detection to $0.07 \mathrm{pM}$ and our optimizations resulting in increased sensitivity, we identified a

429 new distinct sub-population of clinically defined full mutation fully methylated (FM FM) males

430 expressing trace or very low levels, but not absent, FXP. This is the first time, to the best of our

431 knowledge, that an FXP assay has reliably and reproducibly documented very low levels of FXP in FM FM

432 in the context of also identifying subjects with zero FXP. Yet, among FM FM males alone (i.e., excluding

433 males with mosaicism) relationship between FXP and IQ was no longer significant. This suggests that FXP

434 below a specific threshold may no longer be related to intellectual functioning. Replication and further

435 exploration of this result is needed as is a more comprehensive examination of clinical implications of

436 trace production.

437 Our study results must be taken in the context of several weaknesses. First, our sample size of

438 premutation fragile $\mathrm{X}$ carriers and control participants was limited. Given this, a thorough interpretation

439 of premutation FXP levels and their clinical relevance will require future work to enroll larger samples of

440 male and female PMCs to better evaluate PMC FXP expression profiles. Though we can differentiate

441 mean FXP differences between persons with FXS and control subjects in this small sample, increasing

442 our control sample numbers will be important in the future to better define what would be considered a

443 "normal FXP range" in humans. Previous studies used "housekeeping genes", genomic DNA or white 
444 blood cell counts to normalize FXP values in peripheral blood (19). Given the widespread effects on

445 protein synthesis by loss of FXP and limited knowledge about the differential expression of FXP across

446 blood cells, here, we chose not to include a normalization step. Future studies are needed to identify the

447 optimal factors for normalization that are not affected by FXS diagnosis. Additionally, we lack at this

448 time data assessing peripheral FXP expression across development in all patient groups evaluated. It will

449 be important to evaluate for potential developmental shifts in FXP expression across developmental

450 windows. Such information will be imperative to interpreting FXP findings and predicting their clinical

451 impact while also enhancing our understanding of FXS pathology thus aiding potential protein-focused

452 therapeutics development in the future.

In addition, our clinical data presented in this manuscript is limited to IQ alone. Comprehensive,

454 multimodal phenotyping of humans with defined FXP blood levels is needed to better understand the

455 potential FXP-brain-behavior relations that may exist in FXS, PMCs, and in the control group populations.

456 Although we have demonstrated potential associations between FXP expression and general cognitive

457 function in FXS, we need to use more quantitative and direct evaluations of brain function such as high

458 density electrophysiology, neuroimaging, and additional performance based measures to understand

459 potential relationships between FXP expression and human phenotypes. In particular, it will be

460 imperative to increase our subject sample size in the context of deep phenotyping to determine the

461 clinical relevance, if any, of trace versus zero FXP expression in FM FM males with FXS. Given our FXP

462 analysis is a peripheral tissue assay, clear challenges exist regarding whether a blood finding correlates

463 with true brain FXP variance in humans. Comprehensive neurophysiologic, behavioral, and cognitive

464 phenotyping will play a role in addressing this underlying question as will potential future post-mortem

465 study to evaluate FXP across tissues including brain FXP expression analysis. We remain hopeful that

466 enhanced quantification of brain neurophysiology will in the near term enhance our ability to evaluate

467 the impact of FXP expression as measured in blood to brain activity and function. 
469 trace FXP expression in certain males with FM FM FXS. Given the large number of methylation sites on

470 the FMR1 gene, we hypothesize that regular Southern Blot and PCR testing potentially lacks the

471 sensitivity to detect small deviations from true full methylation which could result in some transcription

472 of the FMR1 gene and resultant FXP production. Future in depth molecular study is warranted to further

473 understand human FMR1 methylation patterns while also evaluating FXP expression across patient

474 groups in the context of RNA transcript composition and expression. Such future work may be applicable

475 beyond FXS to understand mechanisms of breakthrough protein expression in genes thought to be 476 completely silenced.

477

\section{Conclusions}

479 FXP can be reliably and reproducibly quantified via peripheral whole blood in humans. Variance in FXP

480 was expected and noted between males and females with FXS as well as between groups of persons

481 with FXS versus PMC and non-FX impacted control groups. We have demonstrated the ability to reliably

482 detect very low or trace levels of FXP in blood. In doing so, we have discovered a cohort of males with

483 FXS clinically characterized at having a FXS full mutation fully methylated FMR1 allele who are

484 expressing FXP in their blood. Consistent with prior work in this field, we observed clinical associations

485 between peripheral FXP expression and general cognitive functioning indicated. Future work is

486 necessary to understand both the clinical relevance and molecular mechanisms of trace FXP expression

487 in a subpopulation of males with FXS. 
491 exchange was optimized due to concerns regarding the efficiency of the bead-antibody coupling

492 reaction.

\begin{tabular}{|c|c|c|c|c|c|c|c|c|c|c|c|c|c|}
\hline \multirow{3}{*}{ Detection Antibody } & \multirow{3}{*}{$\begin{array}{c}\text { Capture } \\
\text { Antibody } \\
\text { Buffer } \\
\text { Exchange }\end{array}$} & \multicolumn{12}{|c|}{ Detection Antibody Concentration } \\
\hline & & \multicolumn{3}{|c|}{$1: 625$} & \multicolumn{3}{|c|}{$1: 1250$} & \multicolumn{3}{|c|}{$1: 2500$} & \multicolumn{3}{|c|}{$1: 3125$} \\
\hline & & $70 \mathrm{pM}$ & $0.55 \mathrm{pM}$ & $0 \mathrm{pM}$ & $70 \mathrm{pM}$ & $0.55 \mathrm{pM}$ & $0 \mathrm{pM}$ & $70 \mathrm{pM}$ & $0.55 \mathrm{pM}$ & $0 \mathrm{pM}$ & $70 \mathrm{pM}$ & $0.55 \mathrm{pM}$ & $0 \mathrm{pM}$ \\
\hline \multirow{3}{*}{ R477 } & \multirow{2}{*}{ - } & 12570 & 409 & 293 & 11038 & 280 & 124 & 10463 & 206 & 69 & 7746 & 135 & 45 \\
\hline & & 12023 & 490 & 213 & 11674 & 331 & 128 & 9843 & 185 & 60 & 9181 & 172 & 47 \\
\hline & + & 12705 & 414 & 195 & 11698 & 320 & 115 & 9978 & 215 & 57 & 9490 & 169 & 83 \\
\hline & & \multicolumn{3}{|c|}{$1: 100$} & \multicolumn{3}{|c|}{$1: 250$} & \multicolumn{3}{|c|}{$1: 500$} & \multicolumn{3}{|c|}{$1: 1000$} \\
\hline & & $70 \mathrm{pM}$ & $0.55 \mathrm{pM}$ & $0 \mathrm{pM}$ & $70 \mathrm{pM}$ & $0.55 \mathrm{pM}$ & $0 \mathrm{pM}$ & $70 \mathrm{pM}$ & $0.55 \mathrm{pM}$ & $0 \mathrm{pM}$ & $70 \mathrm{pM}$ & $0.55 \mathrm{pM}$ & $0 \mathrm{pM}$ \\
\hline \multirow{3}{*}{ ab17722 } & \multirow{2}{*}{-} & 11165 & 196 & 66 & 10263 & 172 & 50 & 9599 & 170 & 46 & 10142 & 155 & 40 \\
\hline & & 11143 & 219 & 63 & 10905 & 180 & 53 & 9396 & 168 & 46 & 9483 & 154 & 39 \\
\hline & + & 13807 & 230 & 59 & 13343 & 200 & 46 & 11804 & 186 & 38 & 11798 & 177 & 39 \\
\hline
\end{tabular}

\section{List of Abbreviations}

496

CV coefficient of variation

497 DBS dried blood spot

498 FM FM full methylated, full mutation

499 FMRP fragile $\mathrm{X}$ mental retardation protein

500 FXP fragile $X$ protein

501 FXS fragile $X$ syndrome

502 ICC intra-class correlation coefficient

503 ID intellectual disability

504 IQ intelligence quotient

505 NS non-significant

506 pM picomolar

507 Pre or PMC premutation carrier

508 SEM standard error of the mean 
509 SB-5 Stanford Binet $5^{\text {th }}$ Edition

510 TDC typically developing control

512 Declarations

\section{Ethics approval and consent to participate}

514 Manuscripts reporting studies involving human participants, human data or human tissue must:

515 All human experiments described in this publication were approved by the Cincinnati Children's Hospital

516 Medical Center Institutional Review Board (IRB \# 2013-7327). All human subjects when able provided

517 informed consent for all study procedures and all subjects under guardianship (minors or adults) had

518 their guardian consent for subject participation with assent, when possible, obtain from the subject him

519 or herself.

520

521 Consent for publication

522 Not applicable

524 Availability of data and materials

525 The datasets used and/or analysed during the current study are available from the corresponding author 526 on reasonable request.

527

528 Competing interests 
529 The authors have no financial interests specifically related to the content of this manuscript. CAE is a

530 current consultant to Confluence, Stalicla, Impel, Forge, and Scioto Bioscience. CAE is the inventor on 531 patents related to the treatment of autism and/or fragile $X$ syndrome.

\section{Funding}

534 This work was funded by the Cincinnati Children's Hospital Research Foundation, by NIMH/NICHD grant 535 U54HD082008 (CAE, PSH), and by NINDS/NICHD grant U54HD104461 (AEB, LMS, PSH, CG, CAE). The 536 funding bodies did not participate in the collection of data, analysis, interpretation of data, or in the 537 writing of the manuscript.

\section{Authors' contributions}

540 AEB and CG participated in experiment design and execution and wrote and edited the manuscript. LMS

541 participated in experiment design and wrote and edited the manuscript. KDC edited the manuscript and 542 participated in data interpretation and design. PSH provided the statistical analyses in the manuscript 543 and edited and approved the manuscript. RM, TA and GL participated in early elements of experimental

544 design and edited and approved the manuscript. CAE conceived the overall experiments, participated in 545 experimental design, data interpretation, and wrote and edited the manuscripts. All authors edited and 546 approved the final manuscript. 
We would like to acknowledge Alyssa Sproles for her valuable insight and direction regarding

immunoassay optimization.

\section{References}

552

553

554

555

556

557

558

559

560

561

562

563

564

565

566

567

568

569

570

571

572

573

574

575

576

577

578

579

580

581

582

583

584

585

586

587

588

589

590

591

592

1. Hagerman RJ. Fragile X syndrome. Molecular and clinical insights and treatment issues. West J Med. 1997;166(2):129-37.

2. Pieretti M, Zhang FP, Fu YH, Warren ST, Oostra BA, Caskey CT, et al. Absence of expression of the FMR-1 gene in fragile X syndrome. Cell. 1991;66(4):817-22.

3. Goldson E, Hagerman RJ. The fragile X syndrome. Dev Med Child Neurol. 1992;34(9):826-32.

4. Darnell JC, Klann E. The translation of translational control by FMRP: therapeutic targets for FXS. Nat Neurosci. 2013;16(11):1530-6.

5. Kaufmann WE, Abrams MT, Chen W, Reiss AL. Genotype, molecular phenotype, and cognitive phenotype: correlations in fragile X syndrome. Am J Med Genet. 1999;83(4):286-95.

6. Tassone F, Hagerman RJ, Iklé DN, Dyer PN, Lampe M, Willemsen R, et al. FMRP expression as a potential prognostic indicator in fragile X syndrome. Am J Med Genet. 1999;84(3):250-61.

7. Rogers SJ, Wehner DE, Hagerman R. The behavioral phenotype in fragile X: symptoms of autism in very young children with fragile $X$ syndrome, idiopathic autism, and other developmental disorders. J Dev Behav Pediatr. 2001;22(6):409-17.

8. Loesch DZ, Huggins RM, Hagerman RJ. Phenotypic variation and FMRP levels in fragile X. Ment Retard Dev Disabil Res Rev. 2004;10(1):31-41.

9. Tassone F, Hagerman RJ, Taylor AK, Hagerman PJ. A majority of fragile X males with methylated, full mutation alleles have significant levels of FMR1 messenger RNA. J Med Genet. 2001;38(7):453-6.

10. Loesch DZ, Huggins RM, Bui QM, Taylor AK, Hagerman RJ. Relationship of deficits of FMR1 gene specific protein with physical phenotype of fragile $X$ males and females in pedigrees: a new perspective. Am J Med Genet A. 2003;118A(2):127-34.

11. Iwahashi C, Tassone F, Hagerman RJ, Yasui D, Parrott G, Nguyen D, et al. A quantitative ELISA assay for the fragile $x$ mental retardation 1 protein. J Mol Diagn. 2009;11(4):281-9.

12. Godler DE, Tassone F, Loesch DZ, Taylor AK, Gehling F, Hagerman RJ, et al. Methylation of novel markers of fragile $X$ alleles is inversely correlated with FMRP expression and FMR1 activation ratio. Hum Mol Genet. 2010;19(8):1618-32.

13. Pretto D, Yrigollen CM, Tang HT, Williamson J, Espinal G, Iwahashi CK, et al. Clinical and molecular implications of mosaicism in FMR1 full mutations. Front Genet. 2014;5:318.

14. LaFauci G, Adayev T, Kascsak R, Brown WT. Detection and Quantification of the Fragile X Mental Retardation Protein 1 (FMRP). Genes (Basel). 2016;7(12).

15. Willemsen R, Mohkamsing S, de Vries B, van den Ouweland A, Galjaard H, Oostra B, et al. Rapid antibody test for fragile $X$ syndrome. The Lancet. 1995;345(8958):1147-8.

16. Loesch DZ, Bui QM, Grigsby J, Butler E, Epstein J, Huggins RM, et al. Effect of the fragile X status categories and the fragile $X$ mental retardation protein levels on executive functioning in males and females with fragile X. Neuropsychology. 2003;17(4):646-57.

17. Dyer-Friedman J, Glaser B, Hessl D, Johnston C, Huffman LC, Taylor A, et al. Genetic and environmental influences on the cognitive outcomes of children with fragile $X$ syndrome. J Am Acad Child Adolesc Psychiatry. 2002;41(3):237-44.

18. LaFauci G, Adayev T, Kascsak R, Kascsak R, Nolin S, Mehta P, et al. Fragile X Screening by Quantification of FMRP in Dried Blood Spots by a Luminex Immunoassay. The Journal of Molecular Diagnostics. 2013;15(4):508-17. 
593 19. Budimirovic DB, Schlageter A, Filipovic-Sadic S, Protic DD, Bram E, Mahone EM, et al. A

594 Genotype-Phenotype Study of High-Resolution FMR1 Nucleic Acid and Protein Analyses in Fragile X

595 Patients with Neurobehavioral Assessments. Brain Sci. 2020;10(10).

$59620 . \quad$ Shrout PE, Fleiss JL. Intraclass correlations: uses in assessing rater reliability. New York: McGraw 597 Hill; 1971.

598 21. Rosner B. Percentage points for a generalized ESD many-outlier procedure. Technometrics.

599 1983;25(2):165-72.

600 22. Roid GH. Stanford-Binet Intelligence Scales, Fifth Edition. Itasca, Illinois: Riverside Publishing;

6012003.

602 23. Sansone SM, Schneider A, Bickel E, Berry-Kravis E, Prescott C, HessI D. Improving IQ

603 measurement in intellectual disabilities using true deviation from population norms. J Neurodev Disord.

604 2014;6(1):16.

605

606

607 